\title{
Masculinidad y violencia: aproximaciones desde el universo del deporte
}

\section{Masculinity and violence: approaches in the sports'universe}

\author{
Julio César Gonzáles Pagés* \\ Daniel Alejandro Fernández González**
}

\begin{abstract}
RESUMEN
El ejercicio de la violencia ha sido una cualidad asignada socio-culturalmente a los hombres en el decurso de la historia. Estos la han utilizado en función de legitimar el poder que poseen en el marco de las relaciones de género, a partir de la posición hegemónica que detentan respecto a las mujeres. Asimismo, los hombres continuamente están siendo violentos con otros hombres y consigo mismos, como parte de la socialización de sus masculinidades. La violencia es un fenómeno que se expresa en múltiples formas: física, verbal, psicológica, económica. Los escenarios en los que se hace visible son igualmente variados: las relaciones de pareja, en el seno del hogar, la escuela, los medios de comunicación o las prácticas deportivas. Estas últimas en su devenir histórico han estado marcadas por la presencia de episodios violentos en todas las áreas que se vinculan a ellas. Al constituirse el universo del deporte como espacio privilegiado para la validación de un modelo hegemónico de masculinidad, han sido los hombres los grandes cultores de esos episodios de violencia. Combatir la violencia y promover una cultura de paz que revierta la tradicional jerarquía que define las relaciones entre hombres y mujeres es un objetivo esencial en aras de un mundo mejor. Entre los actores e instituciones implicados en ello, la educación, en su sentido más amplio, ha de desempeñar un rol fundamental en la consecución de ese ideal.

Palabras-clave: Masculinidad; Violencia; Género; Deporte; Educación.

* Doctor en Ciencias Históricas, Universidad de la Habana (1999). Coordinador General de la Red Iberoamericana de Masculinidades. Profesor de la Facultad de Filosofía e Historia de la Universidad de La Habana.

${ }^{* *}$ Licenciado en Historia, Universidad de La Habana (2008). Integrante de la Red Iberoamericana de Masculinidades. Profesor del Departamento de Estudios Cubanos del Instituto
\end{abstract} Superior de Arte. 


\begin{abstract}
The practice of the violence has been a quality assigned socially and culturally to the men in the course of history. They have used it to legitimize their power inside gender relations, from the hegemonic position they have in relation to women. Likewise, men are frequently violent with other men and against themselves, a characteristic in socialization process of their masculinities. Violence is a phenomenon expressed in several ways: physically, verbally, psychologically and economically. The scenes where it appears are various: relationships, home, school, mass media or sport practices. Sport practices have been influenced by several violent episodes in all sports' fields. Men have been protagonists of these episodes in the sport universe, a space where the hegemonic masculinity model is legitimized. Fighting against violence and promoting a culture of peace is an essential objective to build a better world and to overturn the traditional hierarchy ruling men and women social relations. Among the actors and institutions working on it, education has a primordial role to reach these purposes.
\end{abstract}

Keywords: masculinity; violence; gender; sports; education.

Para aquellos que estamos comprometidos con alcanzar relaciones entre hombres y mujeres, signadas por la equidad, el respeto y el diálogo, fomentar una cultura de paz que revierta la violencia que subyace en las actuales relaciones de género, se convierte en un objetivo ineludible.

En ese sentido, educar a las nuevas generaciones a través de la comprensión de las múltiples formas que adopta la violencia, la manera en que la construcción social de género se convierte en un catalizador de esta, y en la búsqueda de soluciones necesarias para eliminarla, se ha convertido en un elemento priorizado dentro de nuestro trabajo.

Recordemos que la violencia es una de las problemáticas que afectan a las sociedades del presente, desde sus estructuras más básicas - dígase la familia - hasta aquellas más complejas, como las relaciones entre las naciones. Este fenómeno se hace sentir a lo largo de todo el planeta, sin reparar en latitudes geográficas, diferencias culturales, contextos socioeconómicos, multiplicidad de ideologías, identidades colectivas o en la heterogeneidad de espacios de socialización humanos.

A escala mundial, el fenómeno de la violencia se muestra en constante aumento, a pesar de los múltiples esfuerzos que se realizan en pos de detener esa progresión y del conocimiento tácito sobre la gravedad de las consecuencias que implica este flagelo. 
Visualizar la violencia en los distintos ámbitos de las relaciones humanas no se convierte en una tarea difícil de realizar. De un modo u otro, hombres y mujeres se encuentran sumidos en sus garras, la sufren y la ejercen. En un mundo signado por las inequidades, las desigualdades y las discriminaciones de todo tipo: económicas, raciales, sexuales, de género, de nacionalidad; la violencia se convierte en un eje transversal de las relaciones sociales, utilizada como un vehículo para la obtención y el mantenimiento de un poder que convierte en sujetos dominantes a unos pocos y subordina a la gran mayoría.

Las formas que adopta la violencia son disímiles, cual de ellas más despreciable y capaz de provocar daños irreparables. Desde la que se reconoce como más visible: la violencia física, pasando por la psicológica, la emocional, la económica o la violencia sexual; hasta llegar a otras que sobrepasan el marco de las relaciones entre las personas como es la violencia ambiental, esa que mantiene en vilo a todo el planeta por sus apocalípticas consecuencias. La violencia habita y penetra todos los espacios posibles: calles, escuelas, hogares, zonas urbanas y rurales, espacios deportivos y musicales. Fenómenos como las guerras, tan comunes hoy, la llevan a los mares, océanos, a los cielos.

Como bien señalábamos en una idea anterior, la violencia es el recurso por excelencia al que acuden aquellos que poseen y buscan conservar un poder sobre otros y otras. Violencia y poder van de la mano, forman parte de un sistema de dominación-subordinación que impera en el universo de las relaciones entre las personas, entre hombres y mujeres.

La definición de la violencia masculina no debe aislarse del entendimiento de los procesos y relaciones sociales de género (PINEDA, 2008). En un mundo construido social y culturalmente para perpetuar la posición privilegiada de los hombres en ese sistema; la violencia ha sido el instrumento mediante el cual estos han ejercido su hegemonía, legitimando el carácter patriarcal de sus sociedades. La violencia se convierte así en una cualidad propia de los hombres, indispensable para el desarrollo de un modelo de masculinidad hegemónica, al cual todos los hombres deben aspirar; mientras que a las mujeres les están vedadas todas aquellas conductas y patrones que lo conforman.

Según estudios realizados por el experto anglosajón Michael Kimmel, ser masculinos presupone no ser femeninos, o sea, no ser como las mujeres. Kimmel expone cuatro aspectos centrales que existen en el imaginario que tienen los hombres acerca de lo que significa ser masculino.

Al enunciar el último de estos elementos afirma que el hombre debe: "mantener una posición de agresividad y violencia física y psicológica activa todo el tiempo" (KIMMEL, 1997, p. 51). Se tiene que demostrar a otros hombres, a las mujeres, ancianos y niños, el empleo de la agresión física o verbal como cualidad indispensable de hombría y poder masculino. 
Ejercer la violencia es una cualidad que se les impone a los hombres. Forma parte del proceso de construcción de su masculinidad, de las características que los han de definir como hombres en sí, varones hegemónicos. La palabra masculinidad ha sido construida históricamente bajo esos cánones y preceptos, por lo que solo nombrarla ya denota superioridad, fuerza y violencia.

Está inscripta de esa manera en las disposiciones del inconsciente de los hombres y de las mujeres, como bien refiere Pierre Bordieu (BORDIEU, 1999). No cabe dudas que se trata de un conjunto de ideas socio-ideológicasculturales que se han encargado de preservar la hegemonía masculina como centro de poder.

Desde pequeños los hombres son conducidos a la asunción de los patrones conductuales asociados al ser masculino, varón, macho. A los varones se les enseña que para ser hombres deben controlar el mundo y lo primero que deben controlar son a ellos mismos y a las mujeres que lo rodean. El asumir la violencia como parte intrínseca de su identidad y de ese proceso, ocupa un espacio primordial. Las dinámicas del proceso formativo que se les imponen, encierran todo el tiempo la asimilación de conductas violentas, agresivas.

En todos aquellos espacios de socialización que actúan como agentes definidores de lo que deben ser los hombres, la violencia es partícipe directa. Todo lo que los rodea va encaminado a reforzar ese proceso: tanto la familia como la escuela, la radio, la televisión, la interacción con otros niños.

Hombres y mujeres comienzan a ocupar lugares antagónicos en este proceso de construcción y socialización de género. Las normas, valores y estereotipos que ambos aprenden y aprehenden desde la infancia como parte de dicha socialización implican que los niños tienen que acoger la violencia como una normativa imprescindible de cumplir.

Las niñas, por su parte han de alejarse de todas aquellas conductas y espacios que tiendan a fomentar esa cualidad masculina. Ellas están "diseñadas" para cultivar cualidades que difieren totalmente de las asignadas al género masculino. Deben ser amorosas, sentimentales, tiernas, dóciles, débiles.

La violencia se convierte en requisito indispensable para competir, para ser fuertes y activos, para detentar un poder, en fin, para dominar no solo a las mujeres, sino también a otros hombres. Resulta lógico entonces que sea un arma esencial para determinar las relaciones de dominación-subordinación que se establecen en el seno de su género.

Aquellos hombres que no sepan o no logren desarrollar su condición violenta dentro de las relaciones de género, comienzan a ser estereotipados peyorativamente, asociados a lo femenino, discriminados, puesta en duda su masculinidad. 
Al ser discriminados además por otras diferencias ya sean raciales, clasistas, económicas o de orientación sexual se van alejando sus opciones de alcanzar el modelo hegemónico de masculinidad que impone su contexto; comienzan también a ser subordinados, lo cual no los exime de que mantengan un poder y que sean violentos con las mujeres. Al contrario, muchas veces contribuye a reforzar esas actitudes hacia las mujeres que los rodean.

En los momentos actuales donde varios autores coinciden en señalar la existencia de una crisis de la masculinidad, a partir de los avances logrados por los movimientos feministas, con sus lógicas consecuencias para el orden tradicional de género; y a los cambios socio-económicos y culturales que se han sucedido en nuestra "aldea global", con su consecuente pérdida de poderes y privilegios (OLAVARRÍA, 2009; GÁLVEZ, 2009); la salida que escogen muchos varones para demostrar su angustia ante este hecho, es aumentar el ejercicio de episodios violentos contra sus parejas, sus hijos e hijas u otras personas cercanas o no.

Ya lo hemos enunciado. Los hombres ejercen constantemente la violencia contra las mujeres, contra otros hombres. Al mismo tiempo, la presión constante que significa poder reunir todos aquellos requisitos que se consideran indispensables para demostrar la posesión de una hegemonía masculina - lo cual resulta tan complejo como casi imposible de cumplir - condiciona que sobre sí mismos los hombres practiquen la violencia.

Desde el mismo instante en que se ven obligados a suprimir todos esos sentimientos y emociones asociados a lo femenino; los momentos en que son conducidos en su socialización con otros hombres a alcanzar metas que se saben impedidos de realizar; o aquellas situaciones en que por la necesidad de demostrar su masculinidad ponen en peligro su integridad física; los hombres están aplicando sobre ellos mismos las más diversas formas de violencia.

Citar o mostrar ejemplos de cómo se manifiestan en las realidades cotidianas de las sociedades presentes, esta "tríada de la violencia”, definida así por uno de los grandes teóricos de las masculinidades (KAUFFMAN, 1997), pudiera conducirnos a escribir una lista tan extensa como la distancia que nos separa del más alejado planeta del sistema solar. Por ello vamos ubicarnos en el contexto de uno de los espacios de socialización por excelencia de los seres humanos: el universo de las prácticas deportivas; mostrando como el deporte puede convertirse en un catalizador de la violencia en cualquiera de sus variantes.

Históricamente el universo deportivo se ha comportado como un terreno de legitimación y recreación de las relaciones sociales establecidas en los más diversos contextos epocales, geográficos y culturales. Superado desde hace décadas en su definición más primigenia de “....sistema de competiciones físicas que buscan medir y comparar las actuaciones del cuerpo humano concebido como 
polémica siempre perfectible..." (BROWN, 1968, p. 44); se ha convertido en un espectáculo donde convergen y se expresan fenómenos y aspectos sociales como la violencia, las conciencias e identidades colectivas, las relaciones raciales y de género, la corrupción; en un campo donde se interconectan y tienen lugar relaciones económicas, comerciales y se validan intereses políticos propios de las sociedades modernas.

Concebido como un espacio esencialmente diseñado para los hombres, el mundo del deporte, no ha podido desprenderse de los discursos y realidades que subyacen en las dinámicas de las relaciones de género que han regido - y lo continúan haciendo - el decurso de las sociedades a escala planetaria (DUNNING; MAGUIRE, 1997; FULLER, 1997; SILVA; BOTELHO; QUEIRÓS, 2006).

A pesar de los sostenidos avances alcanzados por la mujer, el deporte se ha mantenido como un espacio construido, practicado, disfrutado y regulado mayoritariamente por los hombres. El que ellas se hayan incorporado de manera oficial a la práctica de casi todas las disciplinas, que ocupen su lugar en las gradas como aficionadas o fanáticas e incluso participen de las ganancias económicas que deja la actividad atlética, no ha cambiado el modelo de hegemonía masculina que impera en el universo deportivo.

La práctica deportiva lleva implícita siempre la presencia de conductas y características propias del modelo de masculinidad hegemónica impuesto, cultural e ideológicamente, por esos grupos de hombres que detentan el poder social en cualquier contexto. Hablamos de la fuerza física, la agresividad, la potencia, las habilidades y por supuesto las conductas y acciones violentas en las que subyace la validación del ideal del varón hegemónico, la reafirmación de la detención del poder en las fronteras de lo masculino.

Particularmente, la violencia ha sido una cualidad históricamente asignada a los hombres, utilizada en función de alcanzar dicho poder y legitimar su posición dentro de la relación de dominación-subordinación que se establece. El universo deportivo no es la excepción en cuanto a ello.

Resulta lógico entonces que en aquellas mujeres que participan de este espacio, la sociedad "incorpore" a sus vidas esos códigos y patrones conductuales masculinos, ante la supuesta imposibilidad de colegiar su femineidad en ese mundo, inclusive esas que practican muchas de sus disciplinas, calificadas como violentas e hipermasculinizadas, van a parar mayoritariamente a ese banco de acusadas de "varoniles".

Otra arista, muestra a aquellos hombres - particularmente en la niñez y la adolescencia - que no logran insertarse y demostrar sus éxitos en la práctica deportiva, provistos y estereotipados en muchas ocasiones, de un aura femenina que inclusive llega a poner en duda el criterio homofóbico de "correcta 
orientación sexual”.

Evidentemente existe una relación basada en la inequidad cuando hablamos del vínculo género-deporte; donde la menos beneficiada no solo es la parte que integra el estúpidamente llamado "sexo débil", sino también aquellos hombres, que al menos en el campo de las disciplinas atléticas, no se logran mostrar como "machos hegemónicos" merecedores de disfrutar del poder social que se pone en juego en este espacio.

En todas las áreas donde el deporte ocupa el centro de la atención: terrenos, graderías, peñas y demás sitios de polémica, puestos laborales, artículos periodísticos, transmisiones televisivas, establecimientos y paredes con imágenes y grafitis, se justifica su existencia como actividad social que tributa al comportamiento, actitudes, costumbres y actividades sociales reservados históricamente para hombres hegemónicos.

Subrayar la inexistencia de fronteras geográficas, culturales, de sistemas, religiosas o de idiomas, en el marco de las dinámicas que adoptan las relaciones entre hombres y mujeres en la esfera del deporte, es un elemento importante para entender el carácter universal de esta problemática. No importa si lo visualizamos en una peña beisbolera de la capital cubana, en un estadio de fútbol ecuatoriano donde se juega un derby local, en el monto de las ganancias que genera una liga profesional europea y su distribución; o si nos trasladamos a un campo de refugiados(as) somalíes, ubicado en su vecina Kenya, donde un grupo de mujeres que juegan voleibol, son discriminadas por no cumplir las indicaciones patriarcales que les impone su religión, la musulmana.

Las inequidades de género, la discriminación y la violencia en cualquiera de las variantes que nos presenta Kauffman en su tríada se manifiestan, particularidades aparte, en una misma dimensión y siguiendo el mismo discurso que proyecta el modelo hegemónico de masculinidad. Ejemplo de ello son los tres escenarios que les presentamos a continuación:

\section{Hombres ejerciendo violencia sobre las mujeres}

Pensemos en la situación que nos muestra un documental realizado por la ACNUR en un campamento de refugiados somalíes situado en Kenya (UNHRC, 2008). Allí, como parte del trabajo humanitario que realiza la ACNUR para mejorar las difíciles condiciones de vida de estas personas, y a partir del establecimiento de una perspectiva de género para su consecución; los miembros 
de esa organización han comenzado a incentivar en las mujeres la práctica de disciplinas deportivas, particularmente el voleibol, como vía para modificar la subordinación y la ausencia de libertades en que las refugiadas se encuentran sumidas por la fuerte estructura patriarcal en que se desenvuelven.

Escudados tras los preceptos que dicta su religión, que enuncian y justifican el papel subordinado de la mujer, su supuesta debilidad, las funciones siempre domésticas que les están asignadas por mandato divino, su exclusión de los espacios públicos; y por supuesto por la renuencia y el temor a permitir que ellas participen de una actividad legitimada por su cultura y su propia religión como exclusiva de los hombres; la gran mayoría de los miembros del campamento se oponen a que las mujeres jueguen voleibol.

Esta situación ha condicionado no solo ese rechazo, sino también la adopción de actitudes y prácticas violentas que todo el tiempo actúan en detrimento de la posibilidad de que estas mujeres, muy jóvenes todas, comiencen a vislumbrar que existe una manera más feliz de vivir - no importa que sea en un campamento de refugiados -, que es posible disfrutar de derechos que son inalienables.

Desde el mismo momento en que esos hombres, o mejor sus padres, hermanos, primos, amigos, varones que comparten sus mismas edades, pretenden negarles el derecho a participar de una actividad social que en su concepción es abierta a todos por igual, se está ejerciendo violencia sobre esas mujeres, al discriminarlas, excluirlas.

No solo las agreden al lanzarles piedras u otros objetos mientras juegan; constantemente las presionan emocional y psicológicamente al catalogarlas como "mujeres de la calle", "renegadas de su religión", "jóvenes que nunca encontrarán un esposo que las acepte"; las obligan a jugar usando todo el vestuario que su religión les impone, a pesar del calor sofocante kenyano. Esto provoca que muchas de ellas, aunque sientan el deseo de poder unirse a las que han decidido ignorar esas agresiones, no puedan dar el paso necesario de romper con el estigma de subordinación histórica, propia de su cultura, "bendecida" por su religión. 


\section{Hombres que ejercen violencia contra otros hombres}

Boca Juniors ante River Plate, la Roma versus Lazio; el Manchester United enfrentando al Chelsea, la selecciones nacionales de Argentina y Brasil rivalizan en un partido clasificatorio al próximo mundial. Cualquiera de los estadios que acogen estos partidos de fútbol puede servirnos como escenario perfecto. Los actores principales no se encuentran precisamente en la cancha, no andan corriendo tras un balón. No son estrellas mundiales, íconos de un nuevo modelo del hombre-triunfo moderno. Sus nombres, apellidos o apodos no son coreados a viva voz por miles de personas: Kaká, Ronaldinho, del Piero, Raúl o Drogba.

Los protagonistas de este momento son reconocidos bajo diversas denominaciones, a tenor con la región o el país donde nos encontremos: hinchas, barras bravas, hooligans, tifosis. Un común denominador los define: la inmensa mayoría son hombres, y se distinguen visiblemente del resto de las personas que acuden a un estadio a disfrutar de un partido de fútbol.

Una de las cualidades que poseen estos grupos de hombres es el ejercicio de la violencia. En un estudio realizado a hinchas de los clubes argentinos Colegiales y Huracán, estos coincidían en señalar entre sus particularidades el uso constante de las prácticas violentas. A su juicio, ponían a disposición del honor del club sus saberes violentos para no ser ofendidos por los adversarios. Ello estaba motivado porque subyacente al encuentro futbolístico se dirimían cuestiones de honor del club y de sus simpatizantes que solo pueden debatirse en el plano de los enfrentamientos (GARRIGA, 2005).

Para Dunning entre las causas que condicionan las conductas violentas de hinchas como esos, se encuentra la relación con códigos propios de un modelo de masculinidad dentro del universo deportivo que: resalta hasta el extremo la rudeza y la habilidad para pelear; es, en ese aspecto, distinto de grado - pero no de clase - de las normas de masculinidad actualmente dominantes en la sociedad en general; y tiende a recibir la constante condena de los grupos socialmente dominantes (DUNNING, 1997).

A partir de la formación de una identidad colectiva respecto a su club o selección nacional de preferencia, los hinchas asumen un compromiso y un sentido de pertenencia hacia sus equipos que en muchas ocasiones se convierte en su principal fuente de satisfacción. Derivado en lógicas confrontaciones locales o nacionales, el ejercicio de la violencia se convierte en un eje transversal de su accionar, que busca legitimar la victoria de su club en las gradas, o en su defecto, "escarmentar" a sus rivales por el revés sufrido.

Los ejemplos que pudiéramos citar acerca de las graves consecuencias que han producido enfrentamientos entre hinchadas pudieran ser muchos. Uno 
mundialmente conocido por la estela de muerte y horror que dejó a su paso fue durante el partido Liverpool-Juventus, en 1985.

En este caso, queremos referenciar algunas ideas que nos comentara la abogada uruguaya Graciela Navarro, en una entrevista para la Red Iberoamericana de Masculinidades. Ante una pregunta sobre la participación de las mujeres como espectadoras en los estadios Graciela responde: "cada vez menos me parece... el ambiente en los estadios se ha tornado tan violento que es bastante difícil para la familia asistir al estadio sin correr algún riesgo. Por lo menos eso es lo que está en la conciencia de todos... vas al fútbol en este momento y no sabes bien que te puede pasar" (FERNÁNDEZ GONZÁLEZ, 2009).

\section{Hombres que ejercen violencia contra sí mismos}

Es verano en Cuba, un grupo de jóvenes se encuentra de camping en el litoral norte del occidente de la isla. Suman once varones que comparten varios espacios de socialización: viven todos relativamente cerca, acuden a la misma escuela, van juntos a fiestas y conciertos, y en los meses donde el calor alcanza sus cotas más altas, la playa y el camping se convierten en sus opciones de preferencia.

Entre ellos se pueden observar varias diferencias. Los hay de todas las "razas": blancos, negros y mestizos; se hace evidente que algunos poseen una calidad de vida superior; la complexión de sus cuerpos es diversa: unos son fuertes, con una musculatura bien desarrollada, otros tienen unas "libritas de más", y dos de ellos asemejan "largas y delgadas varas".

Hay una cualidad por sobre todas que los une. Varios autores reconocen la existencia en jóvenes como estos de una tendencia a celebrar el ejercicio de situaciones de riesgo, la práctica de la violencia, así como el descuido y abuso excesivo de sus capacidades corporales, como parte de la búsqueda de un modelo de identidad masculina victorioso, en correspondencia con lo que se espera de ellos como varones (FIGUEROA, 2005). Estos jóvenes no son la excepción.

En la mañana acuden a uno de los sitios más representativos del lugar. Conocido como "la cueva del pájaro", es una especie de poceta en el litoral rodeada por una elevación rocosa que llega a alcanzar los diez metros de altura. Allí, los rasgos propios de la competitividad entre los varones jóvenes, la validación de su "hombría" al enfrentar episodios de riesgo, se convierten en el non plus ultra de sus juegos. Lanzarse a las profundidades de la poceta desde la altura mencionada es la prueba de fuego. La presencia de chicas y de varones 
con experiencia en esa situación, un factor que los incita a no defraudar.

En ese instante, algunos de esos varones, que por primera vez se enfrentan a este "salto ornamental", se ven impelidos, en una demostración de temeridad, valentía y arrojo, a ejecutar el salto. Ello a pesar de que desconocen que la profundidad de las aguas les puede jugar una mala pasada, que lanzarse con miedo les puede hacer chocar contra las rocas laterales. Hacerlo te coloca en el grupo de los "bárbaros", "los hombres a todas", para los cuales el próximo paso es rivalizar a ver quién efectúa los mejores clavados. Aquellos que no logran vencer sus miedos se convierten en objeto de continuas burlas y descalificaciones como: "cobarde", "estás flojo", "no tienes huevos". La posibilidad de sufrir daños físicos en esta situación se da la mano con la presión psicológica que enfrentan estos jóvenes en ese espacio de ocio.

Agotadas ya todas las opciones de saltos, y diferenciados los "salvajes" de los "flojos", una nueva competencia se fragua. Comienzan a nadar mar adentro buscando llegar a la posición donde las aguas del mar adquieren un azul más intenso. Una vez más la situación descrita en el párrafo anterior se repite. Aquellos varones con menos práctica en la natación, empiezan a quedarse atrás. Los mueve el saber que con solo llegar, no importa cuán demorado sea, son eximidos de ser objeto de esas burlas y descalificaciones.

Por ello a pesar de tragar constantemente "buches" de agua, de sentir cómo el cansancio físico comienza a hacer mella en ellos, lo intentan. Lo peor de todo es, que una vez que lleguen, deberán volver.

En solo dos horas estos jóvenes han dado rienda suelta a la competencia masculina. Varios emergieron victoriosos de ella, su condición de superhombres en ese espacio ha sido legitimada por las chicas que intentarán conquistar en la noche y por aquellos que deberán contentarse con tener sueños donde la situación vivida sufra profundos cambios a su favor.

En el relato de estas experiencias vemos que estos varones asumen un comportamiento que algunos autores definen como "mito del héroe", mediante el cual "...muchos varones aprenden que para poder legitimarse como tales deben ser héroes y tener historias que contar” (FIGUEROA, 2005, p. 52).

$$
* * *
$$

La violencia masculina, sustentada por la ideología patriarcal, es un fenómeno que trasciende lo particular. Decir que es un problema solucionable a corto plazo, sería engañarnos a nosotros mismos. Desde nuestras posiciones 
como actores sociales, podemos comenzar a combatirla en primer lugar, respetando la diversidad genérica, sexual, racial y generacional. Tenemos que ser capaces de tolerar la otredad y despojarnos de esos prejuicios sociales que tanto nos dañan y nos hacen menos personas, porque consumen una parte de nuestro yo individual. Lograr esto constituye una tarea harto difícil, pero el punto de partida está en nosotros mismos.

Por su parte, las autoridades y órganos de poder de la sociedad, podrían apoyar enormemente a combatir los problemas de violencia callejera y doméstica. Podrían contribuir a crear talleres, que se impartan en las escuelas, centros laborales, prisiones, etc. Con esto lograrían enfrentar en parte el problema, haciéndolo público y reflexionando con las personas acerca del daño real que produce a nivel psicológico y social. Servirían como sustento, muchas Organizaciones No Gubernamentales (ONG), que defienden los derechos de la diversidad sexual, las mujeres, los niños, los desvalidos, los inmigrantes, los negros y mestizos.

Es un problema que necesita que lo hagamos visible, porque de ello dependen la felicidad y tranquilidad social de millones de personas en el mundo. Es necesario aprender a deconstruir tales estereotipos patriarcales, que lejos de que pensemos que nos ayudan a prepararnos para enfrentar la vida, nos la hacen más difícil porqué nos obligan a cumplirlos al pie de la letra.

\section{REFERENCIAS}

BORDIEU, Pierre. La dominación masculina. Barcelona: Anagrama, 1999.

BROWN, Jean Marie. Sociología política del deporte. París: Editorial Macrolibros, 1968.

DUNNING, Eric; MAGUIRE, Joseph. Las relaciones entre los sexos en el deporte. Estudios Feministas, Florianópolis: UFSC, v. 5, n. 2, p. 321-348, 1997.

FERNÁNDEZ GONZÁLEZ, Daniel Alejandro. Entrevista a abogada uruguaya Graciela Navarro. Red Iberoamericana de Masculinidades, mar. 2009. Disponible en: <http:// www.redmasculinidades.com>. Acceso en: 20/3/2009.

. Por los caminos del género: algunas reflexiones. Red Iberoamericana de Masculinidades, jun. 2009. Disponible en: <http://www.redmasculinidades.com>. Acceso en: 26/6/2009. 
FIGUEROA, Juan G. Elementos para el estudio de la sexualidad y la salud de los varones integrantes de las Fuerzas Armadas. In: PANTELIDES, Edith; LÓPEZ, Elisa. Varones latinoamericanos. Estudios sobre sexualidad y reproducción. Buenos Aires: Editorial Paídos, 2005. p. 47-80.

FULLER, Norma. Identidades masculinas. Varones de clase media en el Perú. Lima: Fondo Editorial-PUCP, 1997.

GÁLVEZ, Luis Edmundo. Las fisuras del modelo de masculinidad hegemónica y sus efectos sobre la cohesión social. In: ASTELLARRA, Judith (Coord.). Pacto entre los géneros y políticas públicas. Género y cohesión social. Barcelona: Instituto de la Mujer (Ministerio de Igualdad), 2009. p. 16-22.

GARRIGA, José Antonio Lomo de Macho. Cuerpo, masculinidad y violencia en un grupo de simpatizantes del fútbol. Cuadernos de Antropología Social, Buenos Aires: UBA, n. 22, p. 201-216, 2005.

GONZALEZ PAGÉS, Júlio Cesar. Género y masculinidad ¿el otro lado de una historia? Nueva Antropología, México, v. XVIII, n. 61, p. 117-126, septiembre 2002.

GONZÁLEZ PAGÉS, Julio Cesar; RODRÍGUEZ, Carlos Ernesto. Si le pegó fue por algo. Estereotipos de violencia masculina. Red Iberoamericana de Masculinidades, jun. 2008. Disponible en: <http://www.redmasculinidades.com>. Acceso en: 26/6/2009.

KAUFFMAN, Michel. Los hombres, el feminismo y las experiencias contradictorias del poder entre los hombres. In: VALDÉZ, Teresa; OLAVARRÍA, José (Comps.). Masculinidad(es) Poder y crisis, Ediciones de las Mujeres. Santiago: Isis Internacional/ FLACSO, 1997. p. 62-73.

KIMMEL, Michael. Homofobia, temor, vergüenza y silencio en la identidad masculina. In: VALDÉZ, Teresa; OLAVARRÍA, José (Comps.). Masculinidad(es) Poder y crisis. Ediciones de las Mujeres, Santiago: Isis Internacional/FLACSO, 1997. p. 48-63.

OLAVARRÍA, José. Apuntes para la construcción de una agenda pro género que incorpore a los hombres. In: ASTELLARRA, Judith (Coord.). Pacto entre los géneros y politicas públicas. Género y cohesión social. Barcelona: Instituto de la Mujer (Ministerio de Igualdad), 2009. p. 30-44.

PINEDA, Javier. Masculinidades y feminismos. Violencia intrafamiliar en doble vía: negociando identidades masculinas. In: COLOQUIO INTERNACIONAL DE ESTUDIOS SOBRE VARONES Y MASCULINIDADES, 3., 2008, Medellín. Materiales... Medellín, 2008. 1 CD-ROM.

RAMÍREZ, Jacques Paul. Breves apuntes teóricos para acercarse al problema del fútbol, masculinidad y violencia. In: SEMINARIO INTERNACIONAL DE MASCULINIDADES. Ecuador, 2002. 1 CD-ROM. 
PAGÉS, J. C. G.; GONZÁLEZ, D. A. F. Masculinidad y violencia...

SILVA, Paula; BOTELHO, Paula; QUEIRÓS, Paula. Género y deporte: la construcción de feminidades y masculinidades. Revista Digital, Buenos Aires, a.11, n. 96, 2006.

UNHRC. Una oportunidad para el deporte. (versión español). In: DVD de la Agencia de la ONU para los Refugiados, 2008, 23 min. (DVD-ROM).

Texto recebido em 29 de maio de 2009.

Texto aprovado em 10 de julho de 2009. 